\title{
Mathematics Learning Support across a Multi-Campus Institution: A Prototype of Virtual Support
}

Cormac Breen, Dublin Institute of Technology, Dublin, Ireland. Email: Cormac.breen@dit.ie Ciaran O'Sullivan, Institute of Technology Tallaght, Dublin, Ireland. Ciaran. Email: Ciaran.OSullivan@ittdublin.ie

Damian Cox, Institute of Technology Blanchardstown, Dublin, Ireland.Email: Damian.Cox@itb.ie

\begin{abstract}
In this paper, a study on Mathematics Learning Support (MLS) that was undertaken across three institutes intending to form the Technological University for Dublin is outlined. This study consisted of a survey that was circulated to both staff and students in each of the three institutes. The survey had two objectives. Firstly it sought to identify the students' needs for MLS in each of the three institutes. Secondly, it sought to ascertain the preferred method of provision of MLS, on a scale ranging from exclusively online, to exclusively in person. Following on the results of this survey it was decided to prototype a virtual MLS drop-in service. The operational details of the prototyping of this virtual drop-in service and the feedback obtained from the students involved are also presented in this paper.
\end{abstract}

Keywords: Mathematics-Support, Virtual Drop-in.

\section{Introduction}

In recent years an increasing number of students in Irish Higher Educational Institutions (HEls) are taking courses with mathematical and statistical elements. This is in part due to the widespread recognition that mathematics underpins the STEM disciplines and the emphasis placed by the Higher Educational Authority (HEA) on producing graduates who are highly literate in mathematics (Expert Group on Future Skills Needs, 2008, and HEA, 2011). Hand in hand with this increase however has come the so called 'Maths Problem'- that is an apparent decline in the mathematical proficiency of incoming first year students across HEls in Ireland and elsewhere (Gill et al., 2010). In fact, it is widely acknowledged that the absence of a solid foundation in mathematics can be one of the key inhibitors for student progression in higher education (HEA, 2010).

As part of the response to this problem, Maths Learning Support Centres (MLSCs), defined by Lawson et al. (2003) as 'a facility offered to students (not necessarily of mathematics) which is in addition to their regular programme of teaching through lectures, tutorials, seminars, problems classes, personal tutorials, etc.' have been set up in the majority of HEls in Ireland (Gill et al., 2008). In fact a soon to be published study involving 32 Irish HEls has shown that $84 \%$ of these now offer some form of MLS (Clancy et al., 2015). It is therefore clear that MLS has now become an integral part of the higher educational framework.

The prosposed merger of the three institutes intending to form the Technological University for Dublin: The Dublin Institute of Technology (DIT), The Institute of Technology Blanchardstown (ITB) and The Institute of Technology Tallaght (ITTD), represents a significant change in the Irish Higher Educational landscape. It is important that the MLS provision in each of these institutes evolves in a manner that best suits the needs of the students across the three Institutes. The work reported in this paper represents a first step in this process. 


\section{Methodology and Participants}

The initial stage of this study consisted of a survey of both staff and students in each of the institutes. It was decided to survey staff that are involved in teaching mathematics or statistics modules and students who had engaged with some form of MLS. The survey was conducted online and consisted of three main questions:

1. Which discipline do you teach under/are you studying?

2. In your opinion, what are the three main topics that students would require MLS for?

3. Please indicate your preference for how this MLS would be delivered?

For Q3, answers were given on a five point scale, ranging from 'Exclusively Online' to 'Exclusively in Person'. A total of 45 staff responses and 118 student responses were received. The breakdown of responses per institute is given in Table 1. It should be noted here that DIT, with approximately 20,000 students enrolled, is a much larger institute than both ITB and ITTD, with approximately 4,000 students each. Table 3 gives details of the responses to Q1 of the survey.

Table 1. Breakdown of Staff and Student Responses by Institute.

\begin{tabular}{|c|c|c|c|c|}
\hline Institute & DIT & ITB & ITTD & Unspecified \\
\hline No. Of Staff Responses & 16 & 18 & 9 & 2 \\
\hline No. Of Student Responses & 34 & 67 & 16 & 1 \\
\hline
\end{tabular}

Table 2. The disciplines under which the staff who responded teach mathematics and the disciplines the student respondents are studying. (Note that respondents could choose more than one option. The percentages quoted are the percentage of respondents in each group who selected each option.)

\begin{tabular}{|c|c|c|c|c|c|c|}
\hline & Engineering & Mathematics & Business & Science & Computing & Other \\
\hline Staff & $59 \%$ & $27 \%$ & $20 \%$ & $27 \%$ & $17 \%$ & $5 \%$ \\
\hline Student & $49 \%$ & $4 \%$ & $24 \%$ & $6 \%$ & $17 \%$ & $3 \%$ \\
\hline
\end{tabular}

\section{Results of the Survey}

In this section we give the results to Q2 and Q3 of the survey. The results from staff and students are given separately and then compared.

It should be noted that the results for Q2, on the topics that students would most need MLS with, are given just in terms of number of responses, as more than one answer to this question was allowed. Many individual responses to Q2 were received, for clarity it was decided to group these responses into five main areas: Basic Algebra (e.g. logarithms, indices), Calculus, Preliminaries (e.g. fractions, basic numeracy, percentages), Probability and Statistics and an Others category. Topics that were included in the Others category include trigonometry, vectors, Fourier and Laplace transforms, geometry, financial mathematics, linear algebra, among others.

The responses to Q3, on the method of provision of MLS, are given as a percentage of total respondents. 


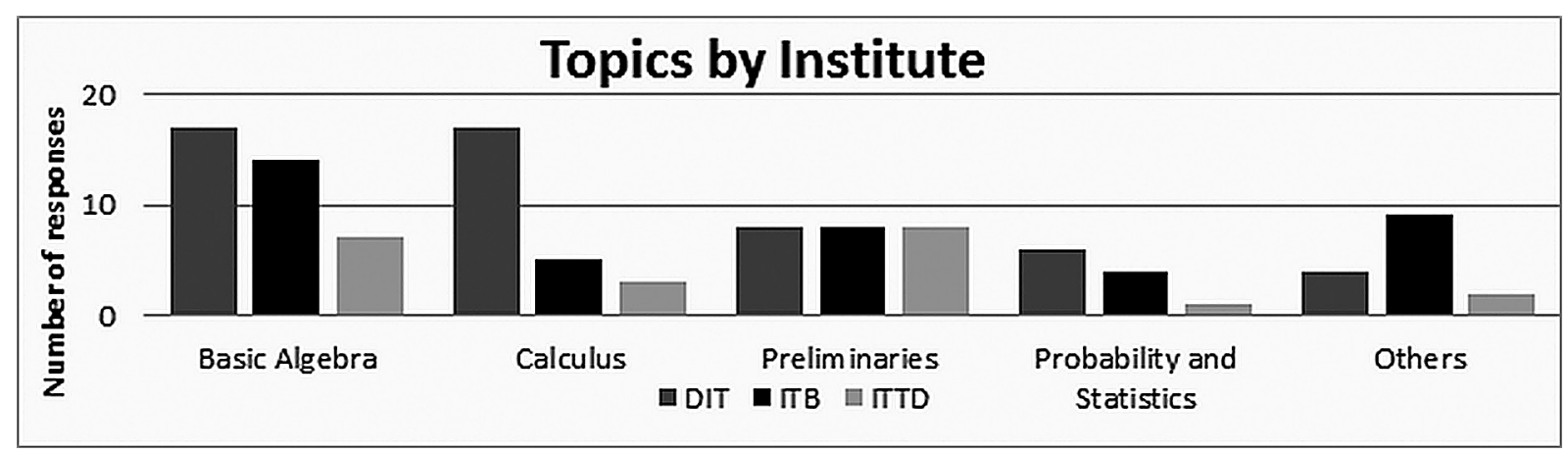

Figure 1. A breakdown of the staff responses to Q2 across each of the Institutes.

Overall the most common topic that staff indicated students would require extra support with was Algebra, followed by Calculus. In DIT the most common topic indicated was Calculus, in ITB it was the Others category and in ITTD the most common topic indicated was Preliminaries.

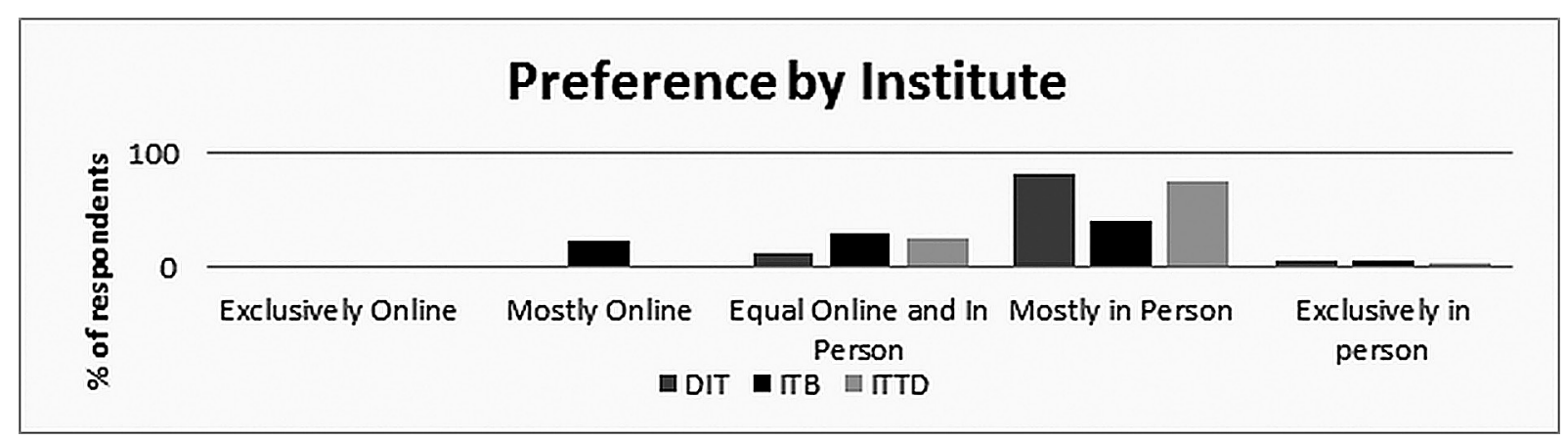

Figure 2: A breakdown of the staff responses to Q3 across each of the Institutes. Preferences are given as a percentage of total responses.

$71 \%$ of total staff surveyed indicated their preference for the delivery of MLS to be provided either mostly or exclusively in person. This was highest in DIT, with $88 \%$ of staff indicating this preference, and lowest in ITB with $47 \%$ of staff indicating their preference to be given mostly or exclusively in person. $24 \%$ of ITB staff would prefer the MLS to be provided mostly online, while $0 \%$ of both ITTD and DIT staff opted for this option. This may be explained by the fact that in ITB some modules are delivered completely or partially online and therefore staff in ITB may be more familiar with this medium of delivery. 


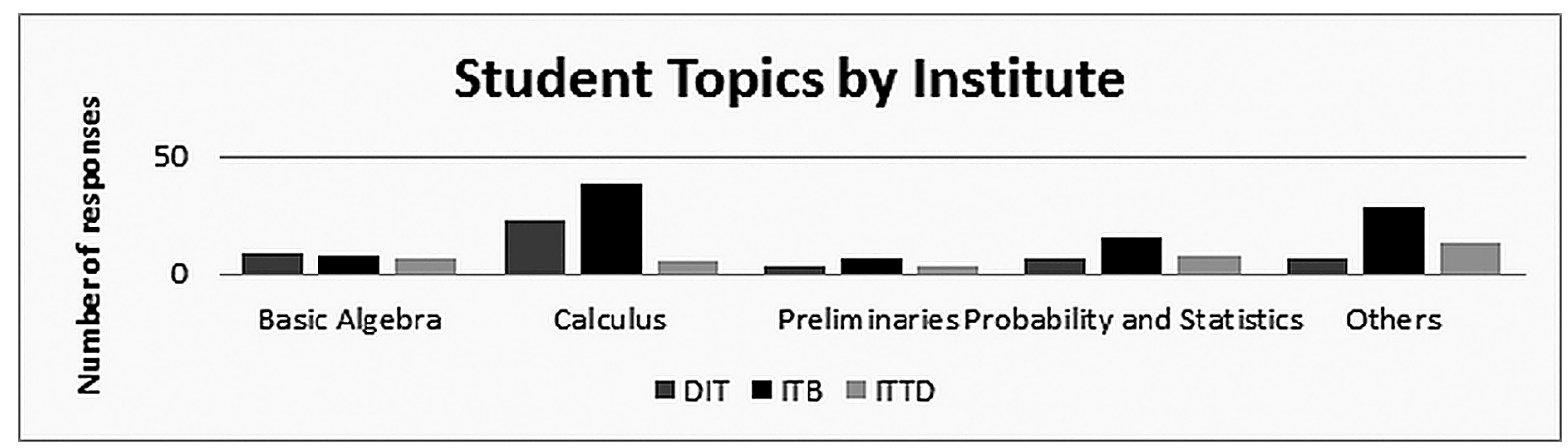

Figure 3. A breakdown of the student responses to Q2 across each of the Institutes.

The most common topic that students indicated overall that they would need extra support with was Calculus; individually Calculus was most commonly chosen in DIT and ITB, while in ITTD the most common topic category was the Others category.

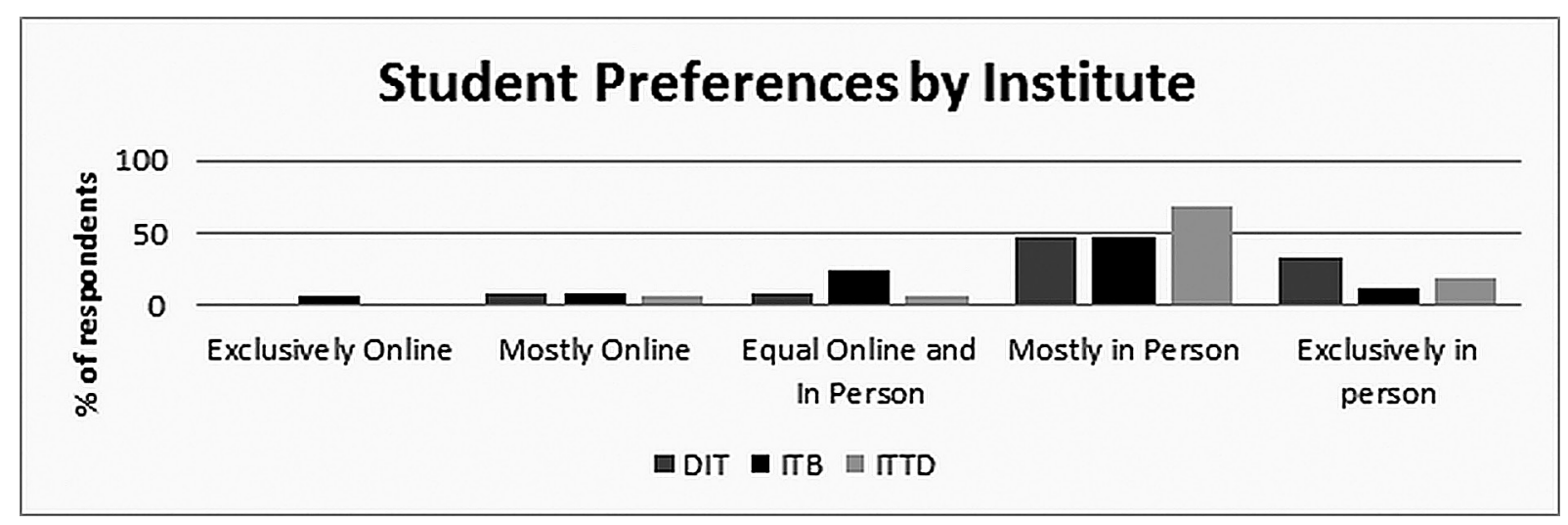

Figure 4. A breakdown of the student responses to Q3 across each of the Institutes

$69 \%$ of total students surveyed indicated that the preference for the delivery of MLS to be provided either mostly or exclusively in person. This was highest in ITTD, with $88 \%$ of students indicating this preference, and lowest in ITB with $60 \%$ of students indicating their preference to be provided mostly or exclusively in person. Again it is worth noting that only ITB students indicated a preference for MLS to be provided exclusively online, which may again be a reflection of their familiarity with online delivery of lecture content.

Comparing the total student and staff responses, we find that the responses to Q3 are mostly in agreement. However it is interesting to note the difference in opinion between the groups in regards to Q2, the topic that they believe students would most need extra support with (see Table 3 for details). 
Table 3: Comparison of the total student and staff responses to Q3

\begin{tabular}{|c|c|c|c|c|c|}
\hline & $\begin{array}{c}\text { Basic } \\
\text { Algebra }\end{array}$ & Calculus & Preliminaries & $\begin{array}{c}\text { Probability and } \\
\text { Statistics }\end{array}$ & Other \\
\hline Staff & $34 \%$ & $22 \%$ & $19 \%$ & $12 \%$ & $13 \%$ \\
\hline Students & $13 \%$ & $37 \%$ & $7 \%$ & $16 \%$ & $26 \%$ \\
\hline
\end{tabular}

A result of particular note is that the most common topic chosen by students was Calculus, while Basic Algebra was the most common topic that staff chose. One possible interpretation of this is as a misconception on the students' behalf, whereby they are struggling with a module involving Calculus but the root causes for this difficulty may lie in issues with Basic Algebra.

\section{Virtual Drop-in Service Prototype}

While the majority of staff and students surveyed had a preference for in person support, there was some desire that some form of MLS be provided online. In a recent large scale report on student engagement with MLS in Ireland, it was found that $83 \%$ of students surveyed rated the drop-in service as worthwhile while $56 \%$ of students rated ICT facilitated support as worthwhile (O'Sullivan, et al. 2014). Inspired by this and the results of our survey, we decided to prototype a virtual drop-in service, where we would hope to replicate the in-person experience as much as possible in a virtual environment. It should also be noted that in the proposed merger both ITB and ITTD will remain in their respective campuses, therefore the development of effective ICT facilitated support, such as a virtual drop-in service, will be an important issue in any future merged entity.

In order to implement this, three Wacom Intuos Tablets were purchased (see Figure 5), one for use in each institute, and the Adobe Connect software package was used. It should be noted here that the Adobe Connect software package was chosen simply because there the institutes involved possess a licence for this package. Another equivalent package, such as Blackboard Collaborate, would also have been sufficient for our purposes. The key feature required of any particular software package is that it can be used to mimic the in-person drop-in experience as closely as possible. This includes a facility to simultaneously have a two way conversation and to share a whiteboard, upon which all participants can write. In keeping with the spirt of collaborative learning, a single Adobe Connect session is run for all students involved in each trial. This provides the opportunity for students to learn from each other as well as from the academic member of staff. With Adobe Connect, students were also able to have screenshots of the shared whiteboard sent to their email, which they could then use for their own independent learning at a later date. It is possible for the entire session to be recorded, however for this first prototype it was decided to only allow the students to take snapshots of the whiteboard.

The first trial involved staff members from each of the institutes; this was then followed by two trials involving seven students in total. The first of these involved students in ITTD receiving support from a staff member in DIT, and the second involved students in ITB and a staff member in ITTD. For these trials the use of tablet and laptops was supervised by the lecturers on each site who were participating (i.e. the three named authors). The tablets were securely stored by the authors in-between sessions.

In the event of rolling out the approach to a larger scale pilot it is envisaged that a staff member involved in learning support (but not necessarily someone capable of providing Mathematics support) would be tasked with setting up the tablet and microphones as well as ensuring their proper use. For example, an administrator at the support centre could be trained to set up the 
tablet etc. and would then be on hand to ensure that any technical issues that arise during the session could be resolved in a timely manner.

After the trials were completed, feedback was sought from the students, including their opinions on the advantages and disadvantages of the virtual drop-in over the traditional experience and any suggestions they may have on improving the virtual drop-in experience.

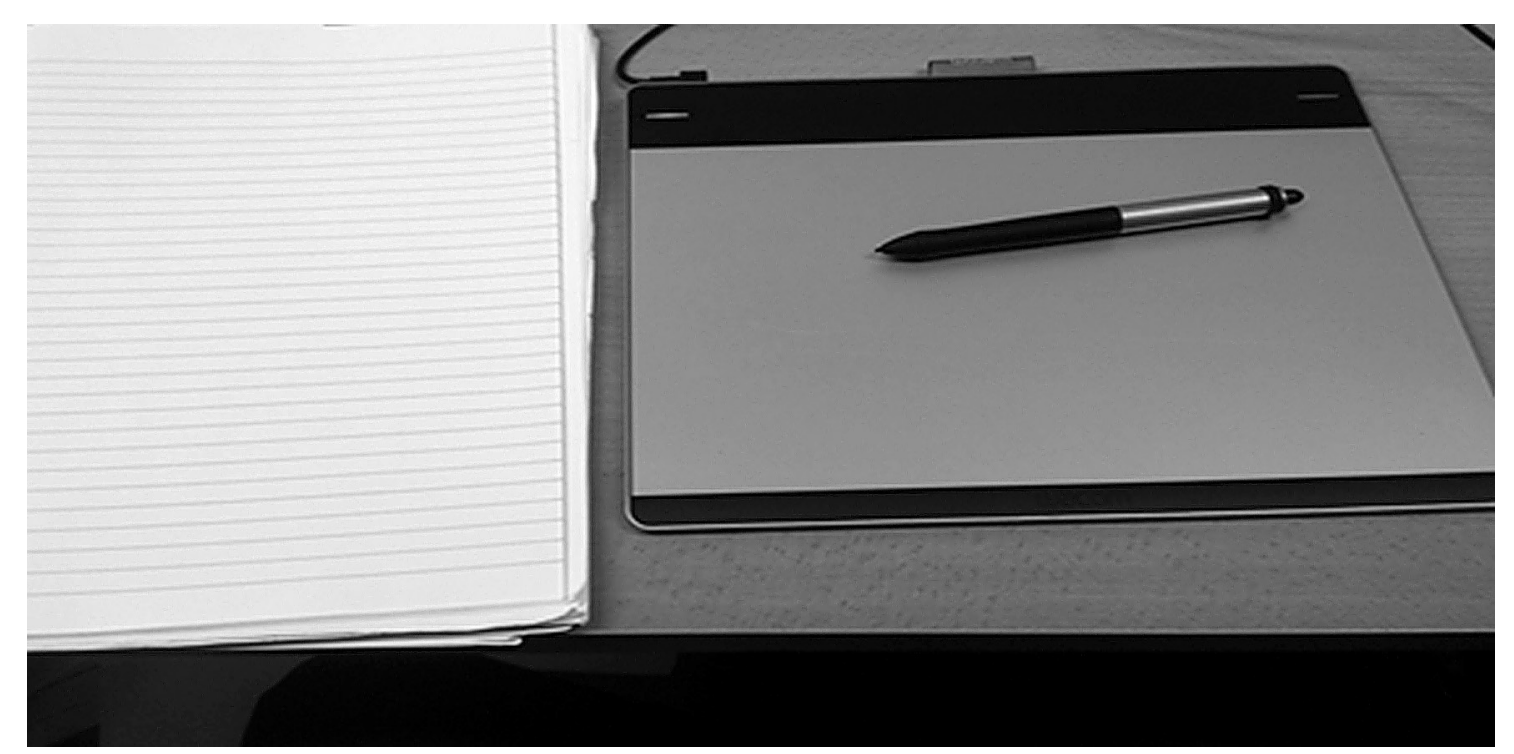

Figure 5. An image of one of the Wacom Intuos Tablets used in this project. An A4 page is included so the relative size of the tablet can be observed.

Overall students were positive towards the concept of the Virtual Drop-in service. However, they felt that the technical issues that arose during the trials, such as feedback and slow connection issues, would need to be addressed in order for this service to be implemented successfully. A representative selection of the feedback received from the seven students involved in the prototype is presented below.

\subsection{Overall Impression}

'I was very impressed, I think it's a great way to help students with Maths questions. Aside from the slow internet feed I think it has great potential and I'm definitely keen to see it up and running'

'Great idea, software was a little clunky and internet connection or lack of was a hindrance, But can easily be taught and overcome'

'It has potential but I feel unless it becomes more streamlined I could see students becoming more frustrated than helped.'

\subsection{Advantages}

'It will help people learn or ask questions easier who are shy or ashamed to do so in class'

'... the virtual drop-in gives a larger scope of time and geographical location.'

\subsection{Disadvantages}

'If the lecturer on virtual drop-in uses different techniques to solve questions as opposed to classroom lecturer, it may be confusing and take longer to help solve a problem' 
'may get overloaded with students who don't bother going to class as they see this system as a substitute for class attendance'

\subsection{Suggestions for Improvement}

'Use external microphone and speakers or headphones to eliminate the echo effect'.

'Use a speed scanner to scan the Example questions or problem sheets faster to save time writing it on the pad'.

'Web cam Web cast with the cameras facing whiteboards on either side of the link'

'A platform for posting question and receiving answers in a timely fashion'

\section{Conclusions}

In this paper we have outlined the results of a survey of staff and students in three institutions soon to form a new merged entity. The majority of staff $(71 \%)$ and students $(69 \%)$ surveyed would like to see MLS provided either primarily or exclusively in person. This is a recurring theme in studies undertaken in Irish Higher Education system, see for example O'Sullivan (et al. 2014) and Clancy et al. (2015), and therefore it is clear that in-person support should remain at the core of MLS provision in Irish HEls.

However among those who participated in this survey there was some preference that a portion of MLS be delivered online. As a result of this preference among both staff and students, a prototype of a virtual drop-in service, aligned closely to the in-person experience, was developed and was tested with students and staff from the different institutes. These students were mostly positive towards the concept of virtual drop-in, but had some concerns on the technical side of the service. They also offered some useful suggestions on how this service could be improved. Indeed based on the feedback from students involved in the proto-typing, headphones and microphones have been purchased for use in future trials.

A point worth stressing is that technical difficulties, such as slow connection speed, will certainly have a negative effect on students' engagement with any initiatives such as this. In fact, it is the authors' opinion that for the case of virtual drop-in, technical difficulties such as encountered in this prototype would be fatal to the introduction of a virtual drop-in system on a wider basis. In order for this to happen, we believe that there must be a robust technical infrastructure in place, ideally with dedicated technical support on-hand should issues arise, otherwise it is highly likely that students will not engage with the support.

Finally, the issue of providing effective virtual MLS is of importance beyond the scope of the merger discussed in this paper. For instance at the Open University much work has also been done in developing a model for virtual MLS provision. The Open University model follows along similar lines to that described in this paper, using Blackboard Collaborate to offer one-to-one virtual drop-in sessions. In this model, it is envisaged that this virtual drop-in will form part of a suite of online resources, including 'screencasts, forums and dedicated context based online workshops' (Golden, 2015). In the collaborative spirt of the Mathematics Learning Support network, it is important that the sharing of ideas continues so that the best practice for the provision of virtual MLS can be established.

\section{Acknowledgements}

This project was funded by the DIT Learning Teaching and Technology Centre. 


\section{References}

Clancy, M., Cronin, A., Cole, J., O'Shea, D. and Breen, C. (2015). An Audit of MLS in Irish HEls. Proceedings of the CETL-MSOR Conference 2015 (to appear).

Expert Group on Future Skılls Needs (2008). Statement on Raising National Mathematical Achievement. Available via

http://www.skillsireland.ie/media/egfsn090616 statement on activity.pdf (last accessed August 2015).

Gill, O., Johnson, P. and O'Donoghue, J. (2008). An Audit of Mathematics Support Provision in Irish Third Level Institutions. CEMTL (Regional Centre For Excellence in Mathematics Teaching and Learning), University of Limerick. Available via

http://supportcentre.maths.nuim.ie/sites/supportcentre.maths.nuim.ie.mathsnetwork/files/uploads/d ocs/FullAudit small.pdf (last accessed November 2015).

Gill, O., O'Donoghue J., Faulkner, F. and Hannigan A. (2010). Trends in performance of science and technology students. International Journal of Mathematical Education in Science and Technology, 41, 323-339.

Golden, G. (2015). Virtual Mathematics Support at the Open University. Presentation given at the $9^{\text {th }}$ Annual IMLSN workshop. Available via

http://supportcentre.maths.nuim. ie/mathsnetwork/imlsn9abstracts (last accessed January 2016).

HEA (2010). A Study in Progression in Irish Higher Education. Available via

http://www.hea.ie/sites/default/files/study of progression in irish higher education 2010.pdf

(last accessed August 2015).

HEA (2011). National Strategy for Higher Education to 2030. Available via

http://www.hea.ie/sites/default/files/national strategy for higher education 2030.pdf (last accessed August 2015).

Lawson, D., Croft, A.C. and Halpin, M. (2003). Good practice in the provision of mathematics support centres, learning and teaching in mathematics, statistics and operational research. LTSN Maths, Stats \& OR Network. Available via http://www.mathcentre.ac.uk/resources/Good\%20Practice\%20Guide/goodpractice2E.pdf (last accessed August 2015).

O' Sullivan, C., Mac an Bhaird, C., Fitzmaurice O. and Ní Fhloinn, E. (2014). An Irish Mathematics Learning Support Network Report on Student Evaluation of Mathematics Learning Support: Insights from a large scale multi-institution survey. NCE-MSTL. Available via http://supportcentre.maths.nuim.ie/sites/supportcentre.maths.nuim.ie.mathsnetwork/files/uploads/d ocs/IMLSNFinalReportPrePrinters.pdf (last accessed November 2015). 